\title{
S. A. GREIBACH
}

\section{A note on the recognition of one counter languages}

Revue française d'automatique, informatique, recherche opérationnelle. Informatique théorique, tome 9, no R2 (1975), p. 5-12.

$<$ http://www.numdam.org/item?id=ITA_1975_9_2_5_0>

(C) AFCET, 1975, tous droits réservés.

L'accès aux archives de la revue "Revue française d'automatique, informatique, recherche opérationnelle. Informatique théorique » implique l'accord avec les conditions générales d'utilisation (http://www.numdam.org/legal. php). Toute utilisation commerciale ou impression systématique est constitutive d'une infraction pénale. Toute copie ou impression de ce fichier doit contenir la présente mention de copyright.

\section{Numdam}

Article numérisé dans le cadre du programme

Numérisation de documents anciens mathématiques

http://www.numdam.org/ 
R.A.I.R.O.

(9e année, août 1975, R-2, p. 5-12)

\title{
A NOTE ON THE REGOGNITION OF ONE GOUNTER LANGUAGES (*)
}

\author{
par S. A. Greibach $\left({ }^{1}\right)$ \\ Communicated by R. V. BOOK
}

\begin{abstract}
Every on-line one counter language can be accepted by a deterministic Turing machine in time $n^{2}$. The family of deterministic on-line one counter languages is properly contained in the family of realtime pushdown store acceptor languages. Any off-line nondeterministic one counter machine accepts in time $n^{3}$ and space $n^{2}$.
\end{abstract}

Various results have been established for the complexity of recognition of both on-line and off-line pushdown store languages. For example, it is known that context-free languages (on-line one pushdown store languages) can be recognized by determinsitic Turing machines in time $n^{3}[1]$ or in space $(\log n)^{2}$ [2]. It is not known if either of these results is optimal. A context-free language is known whose time or space complexity is the realizable least upper bound on time or space complexity for the whole family of context-free languages [3]. For some special cases, better results are known; the family of linear context-free languages is recognizable by deterministic Turing machines in time $n^{2}$ [4]. Off-line one pda languages can be recognized in space $n^{2}$ and time $n^{4}[5]$.

In this note we examine briefly the special case of one counter languages, both on-line and off-line. The main results are that every on-line one counter language can be accepted by a deterministic Turing machine in time $n^{2}$, and any off-line nondeterministic one counter machine accepts in time $n^{3}$ and space $n^{2}$. To prove the off-line result, we show that context-free grammars generate in linear time and hence on-line pdas always accept in linear time.

$\left({ }^{*}\right)$ The research in this paper was supported in part by the National Science Foundation under Grant GJ-803.

(1) Department of System Science, University of California, Los Angeles.

Revue Française d'Automatique, Informatique et Recherche Opérationnelle $\mathrm{n}^{\circ}$ août 1975, R-2. 
The reader is referred to [6] for formal definitions of counters and Turing machines. We assume that our machines accept by empty counter and final state. On-line machines have a one-way input tape reading from left-to-right, while off-line machines are assumed to have a two-way read-only input tape with endmarkers on both sides. A deterministic machine accepts in time $T(n)$ if each input $w$ accepted by $M$ is accepted within $T(|w|)$ steps $\left({ }^{1}\right)$. A nondeterministic machine $M$ accepts in time $T(n)$ if for each input $w$ accepted by $M$ there is a computation of $M$ on $w$ which accepts in at most $T(|w|)$ steps.

First we establish our result for on-line one counter languages.

Theorem 1. Every on-line one counter language can be accepted by a deterministic Turing machine in time $n^{2}$.

\section{Proof}

First, if $L$ is an on-line one counter language, we can assume that $L$ is accepted by a nondeterministic on-line one-counter machine $M$ which advances its input tape each unit of time and accepts with the counter empty [7], [8]. Thus $M$ certainly accepts in time $n$; in an accepting computation on $w$, the counter never exceeds $|w| / 2$.

We now describe a deterministic Turing machine to accept $L$. Let * be a new symbol and assume that we have an encoding $E$ of subsets of the state set $K$ of $M$. We start at time 0 with entry ${ }^{*} E\left(\left\{q_{0}\right\}\right)^{*}$ on the Turing machine tape where $q_{0}$ is the initial state of $M$. Suppose at time $t$ we have ${ }^{*} E\left(S_{0}\right)^{*} \ldots{ }^{*} E\left(S_{i}\right) * \ldots{ }^{*} E\left(S_{m_{t}}\right) *$ on the working tape and input a (the $t+1$ - st input symbol) to $M$. We go through the $m_{t}$ entries one by one. Entry $E\left(S_{i}\right)$ is replaced by $E\left(T_{i}\right)$ where $T_{i}$ contains all and only those states $q \varepsilon K$ such that for some $l \varepsilon\{1,0,-1\}$ and $p$ in $S_{i-l}, M$ on input a has the option of transferring to $q$ adding $l$ to the counter. For $i=0$ we do not consider $l=1$ and for $i=m_{t}$ we do not consider $l=-1$. Finally, if for some $p$ in $S_{m_{t}}$ and input $a, M$ has the option of transferring to state $q$ adding 1 to the counter, we let $T_{m_{t+1}}$ be the set of all such states $q$ and add $E\left(T_{m_{t+1}}\right)^{*}$ at the end of the tape. Thus we see that at time $t$ entry $E\left(S_{i}\right)$ encodes all states $M$ could be in with counter contents i after reading the first $t$ input symbols. So $w$ is accepted if and only if at time $t=|w|$, the set $S_{0}$ contains an accepting state. Clearly $m_{t} \leqslant t$ and ${ }^{*} E\left(S_{0}\right)^{*} \ldots{ }^{*} E\left(S_{m_{t}}\right) *$ can be updated in time $\mathrm{cm}_{t}$ for some constant $c$. Thus $w$ is accepted or rejected in time

$$
c \sum_{t=0}^{|w|}(t+1)=c \frac{(|w|+1)(|w|+2)}{2}
$$

(1) For a word $w,|w|$ is the length of $w$. 
which is less than $c|w|^{2}$ for $|w|>3$. Hence an input of length $n$ is accepted or rejected in time proportional to $n^{2}$.

The bound of $n^{2}$ is not at all a tight one. To the best of the author's knowledge, it is not known whether there are on-line one counter languages not recognizable in realtime by a deterministic multitape Turing machine. It seems reasonable to conjecture that linear time might suffice.

Using the techniques of Theorem 1 we can establish similar results for time bounded multicounter languages. First we establish a result for multihead finite state machines as Corollary 1 , and then use known connections between these machines and polynomially time bounded multicounter machines to yield Corollary 2.

Consider a $k$-head finite state acceptor; see [10] for precise definitions and details. Such a machine must accept in time $c_{1} n^{k}$ for some constant $c_{1}$. Let us extend the construction of Theorem 1 to use a Turing machine, this time with a $k$-dimensional storage tape. In entry $\left(i_{1}, \ldots, i_{k}\right)$ we place an encoding not only of the states the machine could be in with head $j$ on square $i_{j}$ but also the symbol on square $i_{j}, 1 \leqslant j \leqslant k$. An update cycle takes time at most $c_{2} n^{k}$ for some constant $c_{2}$; at the same time a counter can count up to $c_{1} n^{k}$ update cycles. Thus the machine needs at most time $c_{3} n^{2 k}$ and tape $n^{k}$ for an appropriate constant $c_{3}$. Hence an on-line Turing machine with one dimensional storage takes time proportional to $n^{3 k}$.

Corollary 1. A language accepted by a $k$-head finite state machine can be accepted by a deterministic Turing machine in time $n^{3 k}$.

If a language can be accepted by an off-line nondeterministic machine with $r$ counters in time $n^{k}$, it can be accepted by a $(r k+1)$ - head finite state acceptor [6], [10], [11]. Hence we have :

Corollary 2. A language accepted by an off-line nondeterministic $r$-counter machine in time $n^{k}$ can be accepted by a deterministic Turing machine in time $n^{3(k+1)}$.

In the deterministic one counter case we can do better than Theorem 1 . Given a deterministic on-line pushdown store acceptor (pda), we can construct an equivalent one which advances its input tape whenever it is not erasing the store [9]; this construction takes a counter into a counter. But if a deterministic on-line one-counter machine ever performs more subtractions than it has states without advancing its input tape, it will erase the whole counter. Further for any pair of states $q$ and $q^{\prime}$ there are integers $m\left(q, q^{\prime}\right)$ and $n\left(q, q^{\prime}\right)$ such that it will start in $q$ and complete emptying the counter in $q^{\prime}$ if and only if the counter has contents $x \equiv m\left(q, q^{\prime}\right)\left(\bmod n\left(q, q^{\prime}\right)\right)$. Thus a simulating pda could keep track of the $\bmod n\left(q, q^{\prime}\right)$ congruence of $x$ in its finite state control and instead of erasing put down a new "Begin " symbol. Hence it would $n^{\circ}$ août 1975, R-2. 
operate in realtime (assuming it accepts by final state rather than empty store and final state.) Thus every deterministic one counter language is a realtime pda language; the converse is obviously false as the language $\left\{w c w^{R} \mid w \varepsilon\{a, b\}^{*}\right\}$ shows.

Corollary 3. Any deterministic on-line one-counter language can be accepted in realtime by a pda.

Now off-line one counter languages are a special case of off-line pda languages and hence can be accepted by deterministic multitape Turing machines in time $n^{4}$ [5]. We shall prove (Theorem 3) something stronger, namely that an off-line one counter machine always accepts in time $n^{3}$ and space $n^{2}$. This will follow from a result on derivation lengths in context-free grammars : any context-free grammar produces words in linear time in the sense that for any context-free grammar $G$ there is a constant $k$ such that if $G$ generates a word $w$ then some derivation of $w$ takes at most $k|w|$ steps. Applied to pushdown store acceptors this says that any on-line pda in fact accepts in linear time.

Let us use the following notation for context-free grammars. In a contextfree grammar $G=(V, \Sigma, P, S), V$ is a finite vocabulary, $\Sigma \subseteq V$ is the terminal vocabulary, $S \varepsilon V-\Sigma$ the start symbol, and $P \subseteq(V-\Sigma) \times V^{*}$ a finite set of productions or rules. If $(Z, y) \varepsilon P$, usually written $Z \rightarrow y$, and $u, v \varepsilon V^{*}$,

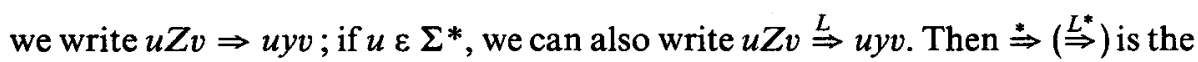
transitive reflexive closure of $\Rightarrow(\stackrel{L}{\Rightarrow})$. The language generated by $G$ is $L(G)=\left\{w \varepsilon \Sigma^{*} \mid S \stackrel{*}{\Rightarrow} w\right\}$. A derivation $Z \stackrel{L^{*}}{\Rightarrow} w$ is called left-to-right.

For a context-free grammar $G=(V, \Sigma, P, S)$, let $v_{G}=\#(V-\Sigma)$ and $k_{G}=\operatorname{Max}\{|y| \mid \exists Z(Z, y) \in P\}\left(^{2}\right)$. In a derivation $\gamma: y_{0} \Rightarrow y_{1} \Rightarrow \ldots \Rightarrow y_{n}$ let $n(\gamma)=n$ and $l(\gamma)=\operatorname{Max}\left\{\left|y_{i}\right| \mid 1 \leqslant i \leqslant n\right\}$. For $Z \in V-\Sigma, w \in V^{*}$, if $Z \Rightarrow W$, let $f_{G}(Z, w)=\operatorname{Min}\{n(\gamma) \mid \gamma: Z \stackrel{*}{\Rightarrow} w\}$ and if $w \in \Sigma^{*}$, let $h_{G}(Z, w)$ be the least $l(\gamma)$ for any left-to-right derivation $\gamma: Z \stackrel{L^{*}}{\Rightarrow} w$.

Theorem 2. Let $G=(V, \Sigma, P, S)$ be a context-free grammar. Let $m_{0}=k_{G}^{v_{g}}$, and $m_{1}=\left(1+\left(k_{G}-1\right) m_{0}\right)$. For $Z \in V-\Sigma$ and $w \in V^{*}$, if $Z \stackrel{\Rightarrow}{\Rightarrow} w$, then

$$
f_{G}(Z, w) \leqslant \begin{cases}m_{0} & w=e\left({ }^{3}\right) \\ \left(v_{G}-1\right) m_{1} & w \in V-\Sigma \\ v_{G} m_{1} & w \in \Sigma \\ \left(v_{G}+k_{G} v_{G}\right) m_{1}|w| & |w| \geqslant 2\end{cases}
$$

(2) For a finite set $A, \#(A)$ is the number of members of $A$.

(3) We use the symnol $e$ for the empty tape; note that $|w|=0$ if and only if $w=e$. 
and if $w \in \Sigma^{*}$

$$
h_{G}(Z, w) \leqslant \begin{cases}\left(v_{G}-1\right)\left(k_{G}-1\right)+1 & w=e \\ {\left[\left(2 v_{G}-1\right)\left(k_{G}-1\right)+1\right]|w|} & |w| \geqslant 1\end{cases}
$$

\section{Proof}

Call a node in a derivation tree expanding if it has two sons each of which has descendent leaves not labeled by the empty string. We proceed by induction on $E(\gamma)$, the number of expanding nodes in a derivation tree of derivation $\gamma: Z \stackrel{*}{\Rightarrow} w$, to show that

$$
f_{G}(Z, w) \leqslant\left(v_{G}+k_{G} v_{G}\right) m_{1} \operatorname{Max}(1, E(\gamma))
$$

and if $w \in \Sigma^{*}$, then

$$
h_{G}(Z, w) \leqslant \begin{cases}\left(v_{G}-1\right)\left(k_{G}-1\right)+1 & w=e \\ {\left[\left(2 v_{G}-1\right)\left(k_{G}-1\right)+1\right]|w|,} & w \neq e\end{cases}
$$

The result follows from the proof of the special case $E(\gamma)=0$, and the fact that $E(\gamma) \leqslant|w|-1$ for $w \neq e$.

First consider $E(\gamma)=0$. There are two cases, $w=e$ and $w \in V$. In the first case consider the tree corresponding to a shortest derivation for $Z \stackrel{*}{\Rightarrow} e$. No nonterminal can appear twice in any path in this tree. Hence each path in the tree has length at most $v_{G}$. In the corresponding left-to-right derivavation $\gamma: Z \stackrel{L^{*}}{\Rightarrow} e, n(\gamma) \leqslant m_{0}=k_{G}^{v_{g}}$ and

$$
l(\gamma) \leqslant k_{G}+\left(k_{G}-1\right)\left(v_{G}-2\right)=\left(v_{G}-1\right)\left(k_{G}-1\right)+1 .
$$

Now suppose $w=A \in V$. Consider the smallest derivation tree for $A$. from $Z$. The path from $Z$ to $A$ has length at most $v_{G}\left(v_{G}-1\right.$ if $\left.A \in V-\Sigma\right)$ and all the brothers of nodes on that path generate the empty string. Hence there is a corresponding derivation $\gamma: Z \stackrel{*}{\Rightarrow} A$, which is left-to-right if $A \in \Sigma$, such that $n(\gamma) \leqslant v_{\mathrm{G}}\left(1+\left(k_{\mathrm{G}}-1\right) m_{0}\right)=v_{\mathrm{G}} m_{1}$, if $A \in \Sigma$ and

$$
n(\gamma) \leqslant\left(v_{G}-1\right)\left(1+\left(k_{G}-1\right) m_{0}\right)=\left(v_{G}-1\right) m_{1}
$$

if $A \in V-\Sigma$.

If $A \in \Sigma$, then in the worst case the left-to-right derivation might have an intermediate string $y_{i}$ containing $v_{G}\left(k_{G}-1\right)$ symbols for the path from $Z$ to $A$ of length $v_{G}$ plus $\left(v_{G}-1\right)\left(k_{G}-1\right)+1$ symbols for the erasing of a left brother of $A$. Hence $h_{G}(Z, A) \leqslant l(\gamma) \leqslant\left(2 v_{G}-1\right)\left(k_{G}-1\right)+1$.

Now suppose that $E \geqslant 1$, that we have shown the result for all $E^{\prime}<E$, $\mathrm{n}^{\circ}$ août $1975, \mathrm{R}-2$. 
and that we have for $Z \stackrel{*}{\Rightarrow} w$ a shortest derivation $\gamma: Z \stackrel{*}{\Rightarrow} w$, such that $n(\gamma)=f_{G}(Z, w)$ and $E(\gamma)=E$. We can divide $\gamma$ into :

$$
\begin{aligned}
& \gamma_{1}: Z \stackrel{*}{\Rightarrow} A \\
& \gamma_{2}: A \Rightarrow x_{1} Y_{1} x_{2} Y_{2} \ldots x_{l} Y_{l} x_{l+1} \\
& \gamma_{i}^{\prime}: x_{i} \stackrel{*}{\Rightarrow} e, \quad 1 \leqslant i \leqslant l+1 \\
& \gamma_{i}: Y_{i} \stackrel{*}{\Rightarrow} w_{i}, \quad 1 \leqslant i \leqslant l
\end{aligned}
$$

where $w=w_{1} \ldots w_{l}, \quad w_{i} \neq e, A, \quad Y_{i} \in V, \quad 1 \leqslant i \leqslant l$, and $l \geqslant 2$ and $l+\left|x_{1} \ldots x_{l+1}\right| \leqslant k_{G}$. (It is possible that $Y_{i}=w_{i}$ for all but two values of $i$.) Thus $A$ labels the first expanding node. Hence $E\left(\gamma_{1}^{\prime}\right)+\ldots+E\left(\gamma_{l}^{\prime}\right)=E(\gamma)-1$.

By the previous results for $E(\gamma)=0$ and the induction hypothesis :

and

$$
\begin{aligned}
& n\left(\gamma_{1}\right) \leqslant\left(v_{G}-1\right) m_{1} \\
& n\left(\gamma_{2}\right)=1 \\
& n\left(\gamma_{i}^{\prime}\right) \leqslant\left|x_{i}\right| m_{0}, \quad 1 \leqslant i \leqslant l+1
\end{aligned}
$$

$$
n\left(\gamma_{1}^{\prime \prime}\right) \leqslant\left\{\begin{array}{lll}
v_{G} m_{1} & \text { if } & E\left(\gamma_{i}^{\prime \prime}\right)=0 \\
\left(v_{G}+k_{G} v_{G}\right) m_{1} E\left(\gamma_{i}^{\prime \prime}\right) & \text { if } & E\left(\gamma_{i}^{\prime \prime}\right) \geqslant 1
\end{array}\right.
$$

Let $r$ be the number of $\gamma_{i}^{\prime \prime}$ with $E\left(\gamma_{i}^{\prime \prime}\right)=0$. Then

and

$$
n\left(\gamma_{1}^{\prime \prime}\right)+\ldots+n\left(\gamma_{l}^{\prime \prime}\right) \leqslant r v_{G} m_{1}+\left(v_{G}+k_{G} v_{G}\right) m_{1}(E(\gamma)-1)
$$

$$
\begin{aligned}
n(\gamma) & \leqslant\left(v_{G}-1\right) m_{1}+1+\left(k_{G}-l\right) m_{0}+r v_{G} m_{1}+\left(v_{G}+k_{G} v_{G}\right) m_{1}(E(\gamma)-1) \\
& \leqslant v_{G} m_{1}+\left(k_{G}-l\right) m_{0}+l v_{G} m_{1}+\left(v_{G}+k_{G} v_{G}\right) m_{1}(E(\gamma)-1) \\
& \leqslant v_{G} m_{1}+k_{G} v_{G} m_{1}+\left(v_{G}+k_{G} v_{G}\right) m_{1}(E(\gamma)-1) \\
& =\left(v_{G}+k_{G} v_{G}\right) m_{1} E(\gamma) .
\end{aligned}
$$

If $w \in \Sigma^{*}$, consider the corresponding left-to-right derivation

$$
\gamma: Z \stackrel{L^{*}}{\Rightarrow} w .
$$

and

$$
\text { Let } s_{1}=\left(v_{G}-2\right)\left(k_{G}-1\right), s_{2}=\left(v_{G}-1\right)\left(k_{G}-1\right)+1 \text {, }
$$

$$
g=s_{1}+s_{2}+2\left(k_{G}-1\right)=\left(2 v_{G}-1\right)\left(k_{G}-1\right)+1 .
$$

In the worst case we have $Z \stackrel{L^{*}}{\Rightarrow} A \alpha$ for $\alpha \neq e$; by our previous reasoning for the case $E(\gamma)=0,|\alpha| \leqslant s_{1}$. Then we have $A \alpha \stackrel{L}{\Rightarrow} x_{1} Y_{1} x_{2} \ldots x_{l} Y_{l} x_{l+1} \alpha$. Recall $l \geqslant 2$ and $\left|x_{1} \ldots x_{l+1}\right| \leqslant k_{G}-l$. Now while we expand each $x_{i}$ the intermediate strings are certainly bounded in length by

$$
\begin{aligned}
& \begin{array}{l}
|\alpha|+\left|w_{1} \ldots w_{i-1}\right|+s_{2}+\left|x_{i} Y_{i} \ldots Y_{l} x_{l+1}\right|-1<|w|+s_{1}+s_{2}+k_{G}-1 \\
\leqslant|w|+g<g|w|,
\end{array} \\
& \text { since }|w| \geqslant 2 \text {, and } g \geqslant 2 .
\end{aligned}
$$


By the induction hypothesis applied to $Y_{i} \stackrel{L^{*}}{\Rightarrow} w_{i}$, while we expand $Y_{i}$ the strings are bounded in length by

$$
\begin{aligned}
& \left|w_{1} \ldots w_{i-1}\right|+|\alpha|+g\left|w_{i}\right|+\left|x_{i+1} Y_{i+1} \ldots x_{l+1}\right| \\
& \quad \leqslant\left|w_{1} \ldots w_{i-1}\right|+g\left|w_{i}\right|+g-1 \leqslant 1+g(|w|-1)+g-1=g|w| .
\end{aligned}
$$

Hence

$$
h_{G}(Z, w) \leqslant g|w| .
$$

In the present instance we need only a simplification of this theorem which we present as a corollary.

Corollary 1. For a context-free grammar $G=(V, \Sigma, P, S)$ there are constants $c_{1}$ and $c_{2}$, with $c_{2}$ independent of $v_{G}$, such that $w$ is in $L(G)$ if and only of there is a left-to-right derivation $\gamma: S \stackrel{L^{*}}{\Rightarrow} w$, with $n(\gamma) \leqslant c_{1} \operatorname{Max}(|w|, 1)$ and $l(\gamma) \leqslant c_{2} v_{G} \operatorname{Max}(|w|, 1)$.

Stated in terms of on-line pdas we have :

Corollary 2. Given an on-line pda $M$ with $q$ states, there are constants $c_{1}$ and $c_{2}$, with $c_{2}$ independent of $q$, such that for all inputs $w, M$ accepts $w$ if and only if there is an accepting computation of $M$ on $w$ taking at most $c_{1} \operatorname{Max}(1,|w|)$ steps in which the pushdown store word never exceeds in length $c_{2} q^{2} \operatorname{Max}(1,|w|)$.

\section{Proof}

In the standard construction of a context-free grammar $G_{M}$ for $M$, if $M$ has $r$ pushdown store symbols, then $v_{\mathbf{G}_{M}} \leqslant r q^{2}$ and a step in a computation of $M$ corresponds exactly to a step in a derivation of $G_{m}$ (see [12]).

For off-line pdas we have the following corollary.

Corollary 3. If $M$ is an off-line pda with $k$ reading heads on its input tape, there is a constant $c$ such that $M$ accepts in space $c n^{2 k}$.

\section{Proof}

If $M$ has $q$ states and acts on an input $w$, we can construct an on-line pda $M_{m}$ with $q(\operatorname{Max}(1,|w|))^{k}$ states which accepts the empty word if and only if $M$ accepts $w$. Since $M_{w}$ uses its pushdown store just as $M$ uses its store on $w$, the space used by $M$ on $w$ is the same as that used by $M_{w}$ on the empty word.

We state the next corollary as a separate theorem.

Theorem 3. If $M$ is an off-line $k$-head one-counter machine, then there is a constant $c$ such that $M$ accepts in space $c n^{2 k}$ and time $c n^{3 k}$.

REMARK. A deterministic $k$-head pda must accept in space $c n^{k}$ for some constant $c$ (or it finds itself in a loop; cf. [5] for details). Hence a deterministic off-line $k$-head one counter machine must accept in space $c n^{k}$ and time $c n^{2 k}$ for some constant $c$. 


\section{BIBLIOGRAPHY}

[1] D. H. YoungeR, Recognition and parsing of context-free languages in time $n^{3}$, Information and Control, (1967), 10, 189-208.

[2] P. M. Lewis, R. E. Stearns and J. Hartmanis, Memory bounds for recognition of context-free and context-sensitive languages, IEEE Conference Record on Switching Circuit Theory and Logical Design, Ann. Arbor, Michigan, 1965, 191-202.

[3] S. A. GreibaCh, The Hardest Context-free Language, SIAM J. Computing, (1973), 2, 304-310.

[4] T. KASAMI, A note on computing time for recognition of languages generated by linear grammars, Information and Control, (1967), 10, 209-214.

[5] A. V. Aho, J. E. Hopcroft and J. D. Ullman, Time and tape complexity of pushdown automaton languages, Information and Control, (1968), 13, 186-206.

[6] P. C. Fischer, A. R. MeYer and A. L. Rosenberg, Counter machines and counter languages, Math Systems Theory, (1968), 2, 265-283.

[7] S. A. GReIBACH, Erasable context-free languages, to appear.

[8] S. Ginsburg and G. F. Rose, The equivalence of stack counter acceptors and quasi-realtime acceptors, J. Computer System Sciences, (1974), 8, 243-269.

[9] S. GinsbuRg and S. GreibaCH, Deterministic context-free languages, Information and Control, (1966), 9, 620-648.

[10] O. H. IbarRa, On two-way multihead automata, J. Computer System Sciences, (1973), .7, 28-36.

[11] S. GreibaCH, Remarks on the complexity of nondeterministic counter languages, to appear.

[12] S. GinsBurg, The Mathematical Theory of Context-Free Languages, McGraw-Hill, New York, 1966. 